\title{
Complex Phase Synchronization in an Array of Oscillators Coupled by Time-Varying Resistor
}

\author{
Yoko Uwate, Student Member, IEEE and Yoshifumi Nishio Member, IEEE
}

\begin{abstract}
In recent years, many people have been trying to develop some applications to information processing by exploiting oscillatory phenomena in neural networks. Bifurcation and stability of equilibrium points in a simple neural oscillator consisting of two neurons have been analyzed in detail. On the other hand, oscillatory phenomena in the simple neural oscillator can be modeled by electrical circuit such as van der Pol oscillators. In this study, we propose a network model of simple oscillators coupled by time-varying resistor, which can explain some interesting complex phenomena observed in a large scale network of neurons coupled by both excitability and inhibitory synapses. By carrying out computer simulations and circuit experiments, we confirm the generation of various interesting phenomena which cannot be observed in simple coupled oscillatory networks.
\end{abstract}

\section{INTRODUCTION}

In recent years, many people have been trying to develop some applications to information processing by exploiting oscillatory phenomena in neural networks. Such neural networks can produce some kinds of phase patterns, and they may be utilized for associative memory or image processing [1]-[4]. Bifurcation and stability of equilibrium points in a simple neural oscillator consisting of two neurons have been analyzed in detail. Further, two neural oscillators coupled by a simple connection have been reported to produce phase synchronization.

On the other hand, oscillatory phenomena in simple neural oscillators can be modeled by electrical circuits such as van der Pol oscillator and BVP oscillator. Studies on synchronization phenomena of coupled oscillators are extensively carried out in various fields, physics [5]-[8], biology [9][10], electrical engineering [11]-[20], and so on. Endo et al. have reported details of theoretical analysis and circuit experiments about some coupled oscillators as a ladder, a ring and a two-dimensional array [13]-[15]. Yamauchi et al. have discovered very interesting wave propagation phenomena of phase states between two adjacent oscillators in an array of van der Pol oscillators coupled by inductors [18][19]. Because many researchers suggest that synchronization phenomena of coupled oscillators have some relations to information processing in the brain, we consider that it is very important to investigate the synchronization phenomena of coupled oscillators to realize a brain-like computer in future application.

In this study, we propose a network model of simple oscillators coupled by time-varying resistor, which can explain

Yoko Uwate and Yoshifumi Nishio are with Dept. of Electrical and Electronic Engineering, Tokushima University, 2-1 Minami-Josanjima, Tokushima, Japan, (phone: +81-88-656-7470; fax: +81-88-656-7471; email: \{uwate, nishio\}@ee.tokushima-u.ac.jp). some interesting complex phenomena observed in a large scale network of neurons coupled by both excitability and inhibitory synapses. We realize the time-varying resistor by switching a positive and a negative resistors periodically. We consider that the positive and the negative effects of the coupling resistor can correspond to the excitability and the inhibitory synapses qualitatively. First, we analyze phase synchronization observed in two van der Pol oscillators coupled by the time-varying resistor as a basic oscillators. Next, we investigate phase synchronization observed in a one-dimensional array of coupled van der Pol oscillators. By carrying out computer simulations and circuit experiments, we confirm the generation of various interesting phenomena which cannot be observed in simple oscillatory networks coupled by resistors. We consider that switching between the excitability and the inhibitory produce the complexity in the network. Furthermore, we carry out computer simulations as changing the timing of the switchings and confirm that the behavior of the whole network becomes more complex.

\section{Coupled Oscillators Model}

Figure 1 shows the circuit model. In this circuit, two identical van der Pol oscillators are coupled by a TimeVarying Resistor (TVR). We have known the synchronization phenomena for the case that the coupling resistor is a simple time-invariant resistor [11][12]. Namely, the in-phase synchronization is stable for a positive coupling resistor, while the anti-phase synchronization is stable for a negative coupling resistor. In this study, we consider the case that the coupling resistance $R(t)$ of the TVR varies with time. We consider that the positive and the negative effects of the coupling resistor can correspond to the excitability and the inhibitory synapses qualitatively. The characteristics of the TVR are shown in Fig. 2. In this study, we consider the case that the duty ratio is fixed as $p=0.5$.

Firstly, the $v_{k}-i_{R k}$ characteristics of the nonlinear resistor are defined as follows,

$$
i_{R k}=-g_{1} v_{k}+g_{3} v_{k}^{3} .
$$

By changing the variables and the parameters,

$$
\begin{gathered}
v_{k}=\sqrt{\frac{g_{1}}{g_{3}}} x_{k}, \quad i_{k}=\sqrt{\frac{g_{1}}{3 g_{3}}} \sqrt{\frac{C}{L}} y_{k}, \quad t=\sqrt{L C} \tau, \\
\epsilon=g_{1} \sqrt{\frac{L}{C}}, \quad \gamma=r \sqrt{\frac{C}{L}}, \quad \omega=\frac{1}{\sqrt{L C}} \omega_{t},
\end{gathered}
$$




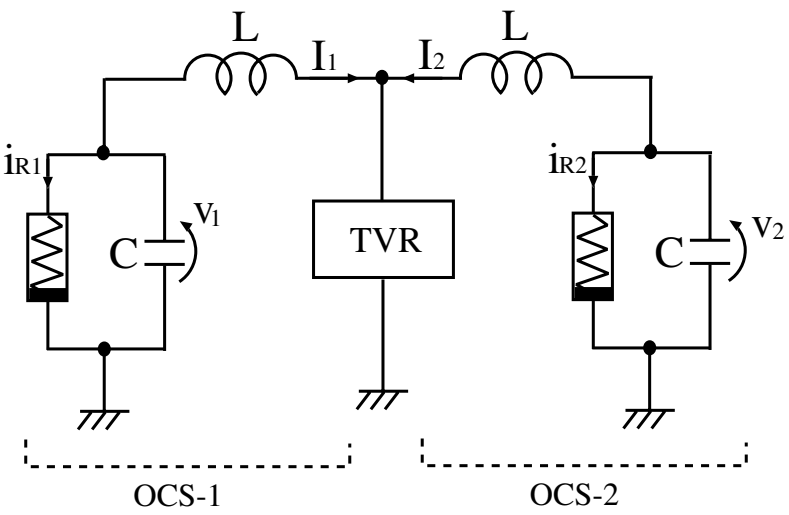

(a) Circuit model (TVR is a Time-Varying Resistor).

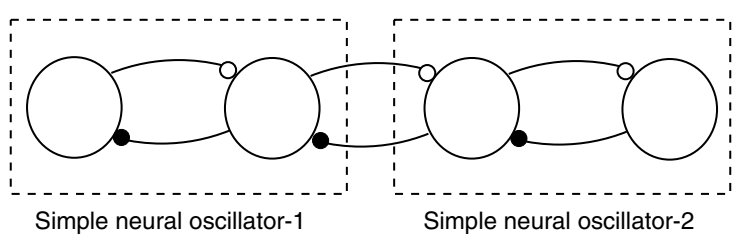

(b) Network topology.

Fig. 1. Coupled oscillators model.

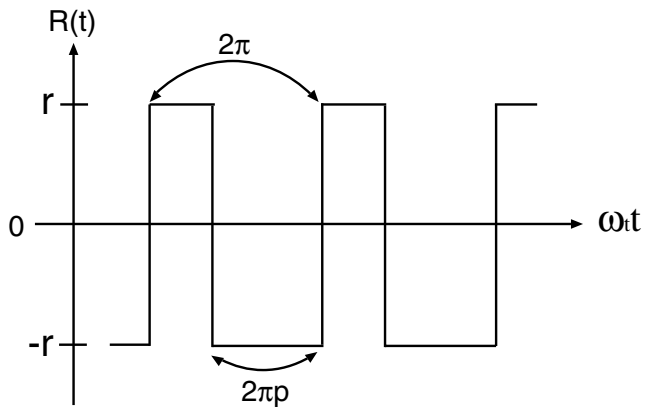

Fig. 2. Characteristics of the TVR.

the normalized circuit equations are given as

$$
\left\{\begin{array}{l}
\frac{d x_{k}}{d \tau}=\epsilon\left(1-x_{k}^{2}\right)-y_{k} \\
\frac{d y_{k}}{d \tau}=x_{k}-\gamma(\tau) \sum_{j=1}^{2} y_{j}
\end{array} \quad(k=1,2)\right.
$$

where the sign of the coupling term changes according to the value of the time-varying resistor.

\section{SYNCHRONIZATION PHENOMENA}

We observed that the two coupled oscillators are synchronized at in-phase or at anti-phase as shown in Figs. 3 and 4. These two synchronization states can be obtained by giving different initial conditions. The parameters of the oscillators model are fixed as $\epsilon=2.6, \omega=1.37$ and $\gamma= \pm 0.1$.

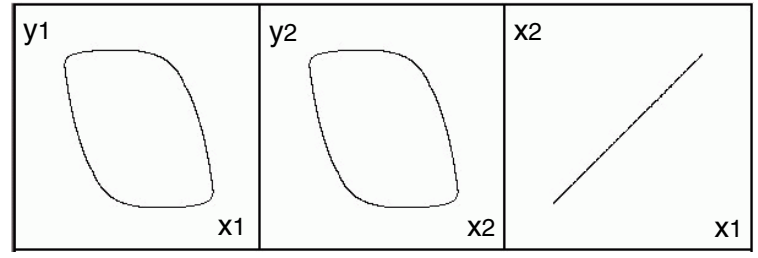

(a)

(b)

(c)

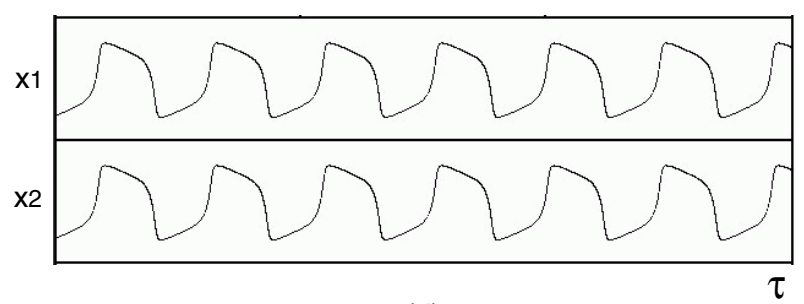

(d)

Fig. 3. In-phase synchronization (computer simulation results). (a) 1st circuit attractor $\left(x_{1}\right.$ vs $\left.y_{1}\right)$. (b) 2 nd circuit attractor $\left(x_{2}\right.$ vs $\left.y_{2}\right)$. (c) Phase difference $\left(x_{1}\right.$ vs $\left.x_{2}\right)$. (d) Time wave form ( $\tau$ vs $x_{1}$ and $\left.x_{2}\right)$.

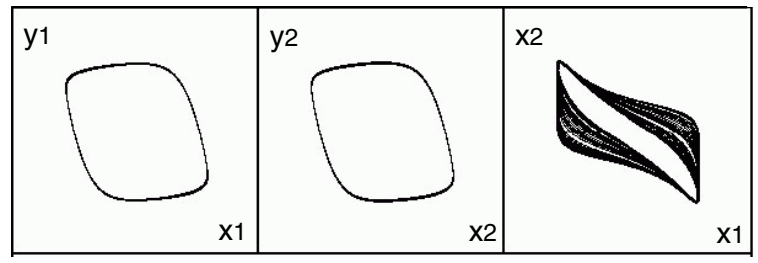

(a)

(b)

(c)

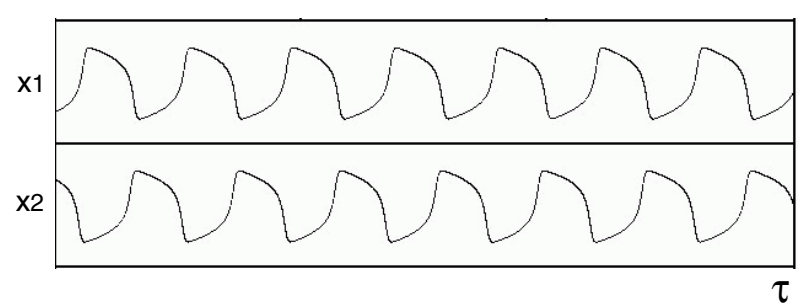

(d)

Fig. 4. Anti-phase synchronization (computer simulation results). (a) 1 st circuit attractor $\left(x_{1}\right.$ vs $\left.y_{1}\right)$. (b) 2 nd circuit attractor ( $x_{2}$ vs $\left.y_{2}\right)$. (c) Phase difference $\left(x_{1}\right.$ vs $\left.x_{2}\right)$. (d) Time wave form ( $\tau$ vs $x_{1}$ and $x_{2}$ ).

We also confirm that the two coupled oscillators are synchronized at in-phase or at anti-phase in circuit experiments as shown in Figs. 5 and 6. In the circuit experiments, the TVR is realized by using an analog switch shown in Fig. 7 [21].

\section{LARGE SCALE OSCILLATORS}

\section{A. Array of Oscillators}

In this section, we consider a ring of oscillators as shown in Fig. 8. In this circuit adjacent two oscillators are coupled by one TVR. We introduce small resistors $r_{m}$ in computer simulations to avoid $L$-loop. By adding the following parameter

$$
\eta=r_{m} \sqrt{\frac{C}{L}}
$$




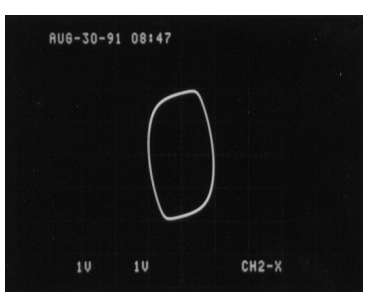

(a)

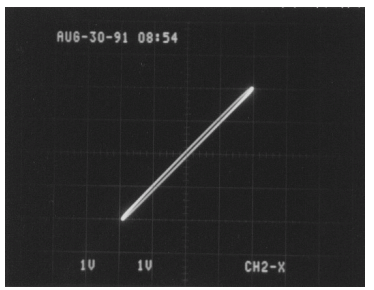

(c)

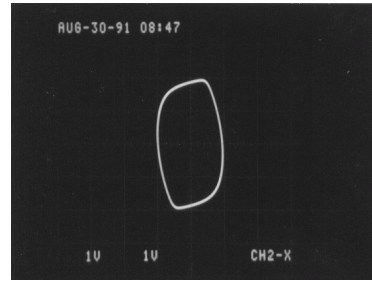

(b)

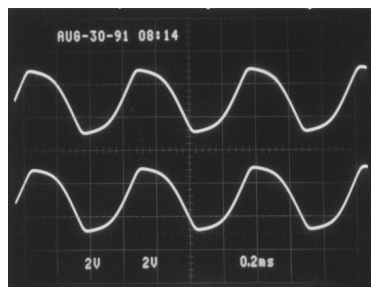

(d)
Fig. 5. In-phase synchronization (circuit experimental results). (a) 1 st circuit attractor ( $v_{1}$ vs $\left.I_{b 1}+I_{a 2}\right)$. (b) 2 nd circuit attractor ( $v_{2}$ vs $I_{a 1}+I_{b 2}$ ). (c) Phase difference ( $v_{1}$ vs $\left.v_{2}\right)$. (d) Time wave form ( $\tau$ vs $v_{1}$ and $v_{2}$ ). $L=10 \mathrm{mH}, C=33 \mathrm{nF}, r=152 \Omega$.

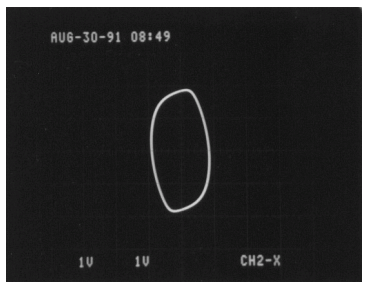

(a)

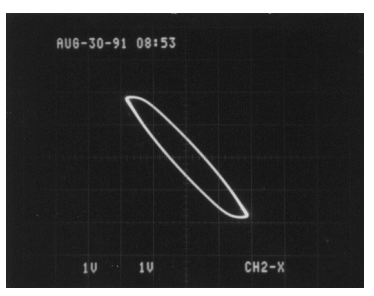

(c)

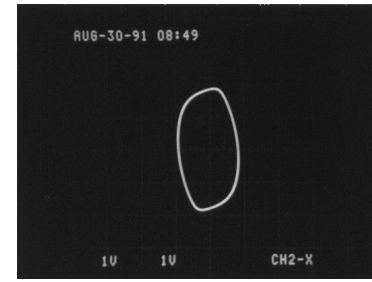

(b)

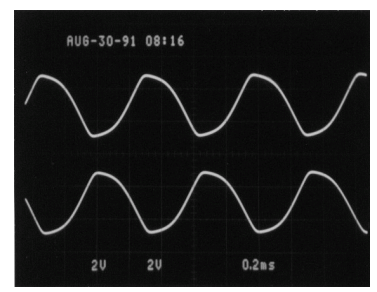

(d)
Fig. 6. Anti-phase synchronization (circuit experimental results). (a) 1 st circuit attractor ( $v_{1}$ vs $I_{b 1}+I_{a 2}$ ). (b) 2 nd circuit attractor ( $v_{2}$ vs $I_{a 1}+I_{b 2}$ ). (c) Phase difference $\left(v_{1}\right.$ vs $\left.v_{2}\right)$. (d) Time wave form ( $\tau$ vs $v_{1}$ and $\left.v_{2}\right)$. $L=10 \mathrm{mH}, C=33 \mathrm{nF}, r=152 \Omega$.

the normalized circuit equations of the array of oscillators are given as

$$
\left\{\begin{aligned}
& \frac{d x_{k}}{d \tau}=\epsilon\left(1-x_{k}^{2}\right)-\left(y_{a k}+y_{b k}\right) \\
& \frac{d y_{a k}}{d \tau}=\frac{1}{2} x_{k}-\eta y_{a k}-\gamma(\tau)\left(y_{a k)}+y_{b(k+1)}\right) \\
& \frac{d y_{b k}}{d \tau}=\frac{1}{2} x_{k}-\eta y_{b k}-\gamma(\tau)\left(y_{a(k-1)}+y_{b k)}\right) \\
&(k=1,2, \cdots, N)
\end{aligned}\right.
$$

where

$$
y_{a 0}=y_{a N}, \quad y_{b(N+1)}=y_{b 1}
$$

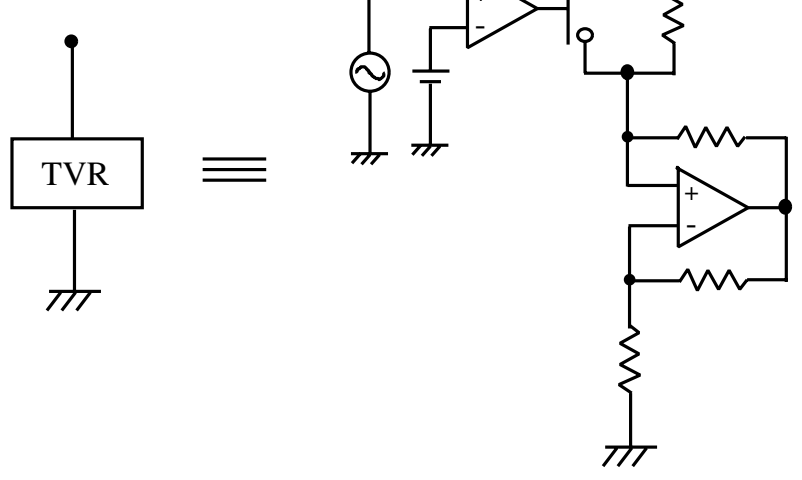

Fig. 7. Circuit realization of the TVR [21].

We can confirm the synchronization phenomena which have not been observed in oscillators coupled by timeinvariant resistor.

\section{B. Even Number Coupling: $N=14$}

Figure 9 shows the computer simulated result for the case of $N=14 . N$ denotes the number of coupled oscillators. We can see that the array of oscillators coupled by TVR are synchronized at in-phase or at anti-phase.

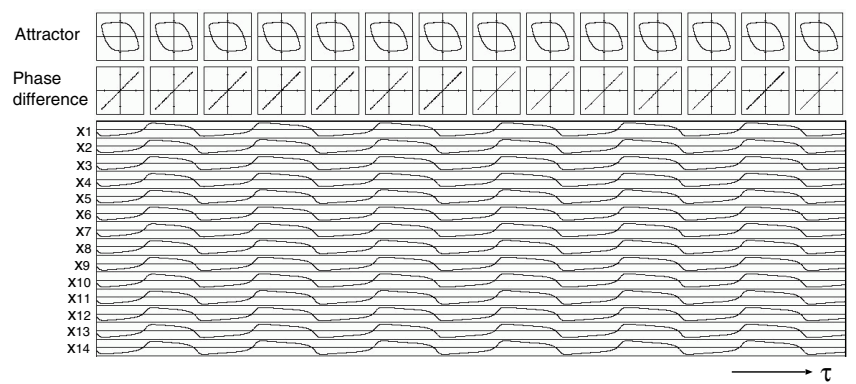

(a) In-phase synchronization.

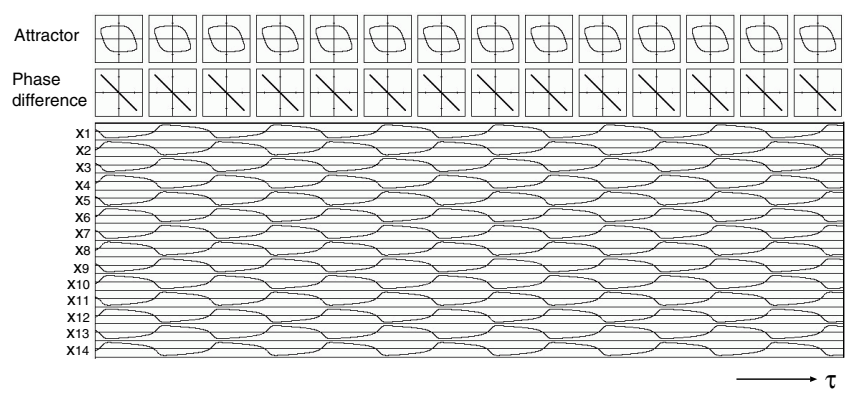

(b) Anti-phase synchronization.

Fig. 9. Computer simulated result for $N=14$. $\varepsilon=2.6, \omega=1.37, \gamma=$ (0.4 or -0.12$), \eta=0.01$. Upper figures: $x_{k}$ vs $y_{a k}+y_{b k}$. Middle figures: $x_{k}$ vs $x_{(k+1)}$. Lower figures: $\tau$ vs $x_{k} . k=1,2,3, \ldots, 14$. 


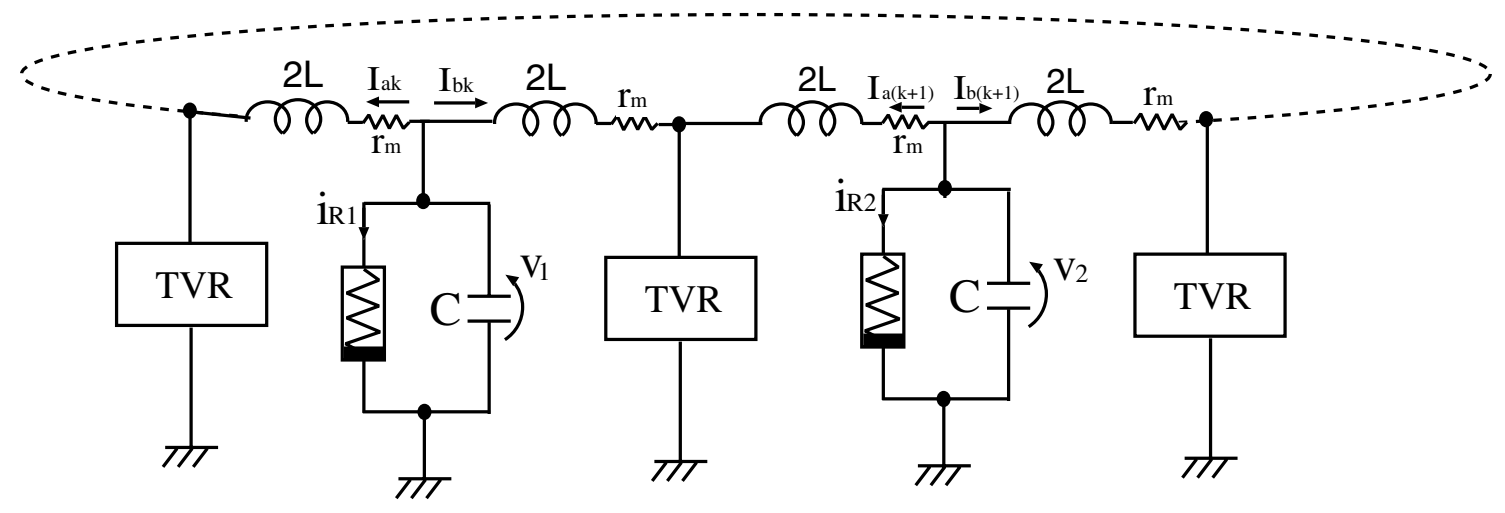

(a) Ring of oscillators.

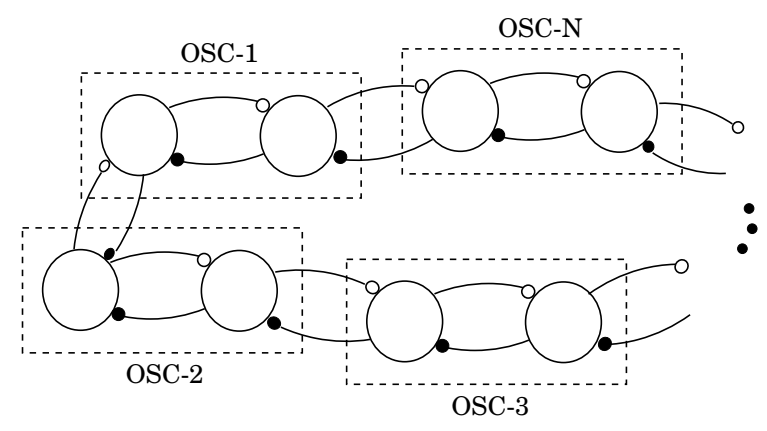

(b) Network topology.

Fig. 8. Ring of circuits model.

\section{Odd Number Coupling: $N=15$}

Figure 10 shows the computer simulated result for the case of $N=15$. We can see that the array of oscillators coupled by TVR are synchronized with in-phase (Fig. 10(a)). However, the adjacent oscillators are almost synchronized with anti-phase as shown in Fig. 10(b). Because, the boundary condition is the ring structure, the phase difference between the adjacent oscillators in not around $\pi$. Namely, in this case 15-phase synchronization are observed.

\section{Wave Propagation Phenomena}

In this section, wave propagation phenomena observed from the 15-array of oscillators coupled by TVR are investigated. Figure 11 shows one example of wave propagation phenomena. In this figure, the vertical axis is the sum of the voltages of adjacent oscillators and the horizontal axis is time. White regions in the diagram correspond to the states that the sum of the voltages is close to zero, namely the adjacent two oscillators are synchronized at anti-phase. While, black regions correspond to the state the sum of the voltages has large amplitude. We can see that the adjacent two oscillators are synchronized at in-phase in the brack regions from Fig. 11.

\section{Complex Phenomena}

We carry out computer simulations as changing the timing of the switchings. Some examples of complex phenomena are shown in Fig. 12. From these figures, various kinds of complex synchronization are confirmed. We consider that switching between the excitability and the inhibitory produce the complex phenomena such as pattern formations.

\section{CONCLUSIONS}

In this study, we proposed a network model of simple oscillators coupled by time-varying resistor, which can explain some interesting complex phenomena observed in a large scale network of neurons coupled by both excitability and inhibitory synapses. By carrying out computer simulations and circuit experiments, we confirmed the generation of various interesting phenomena which cannot be observed in simple oscillatory networks coupled by resistors. We consider that switching between the excitability and the inhibitory produce the complexity in the network.

\section{REFERENCES}

[1] D. Wang, "Emergent synchrony in locally coupled neural oscillators," IEEE Trans. Neural Networks, vol.6, no.4, pp.941-948, Apr. 1995.

[2] S. Campbell and D. Wang, "Synchronization and desynchronization in a network of locally coupled wilson-cowan oscillators," IEEE Trans. Neural Networks, vol.7, no.3, pp.541-553, Mar. 1996. 


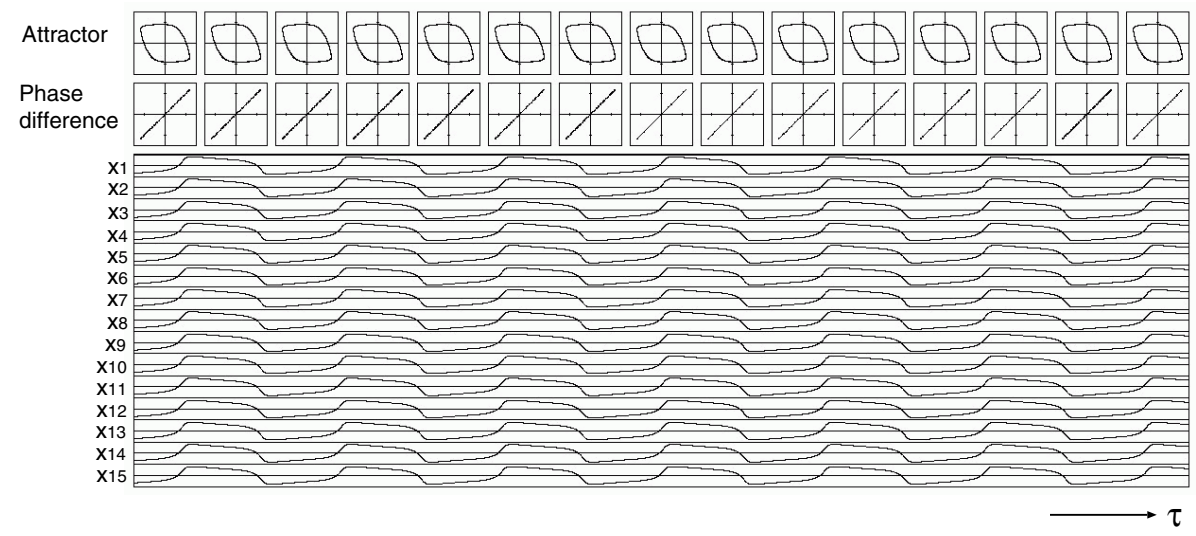

(a) In-phase synchronization.

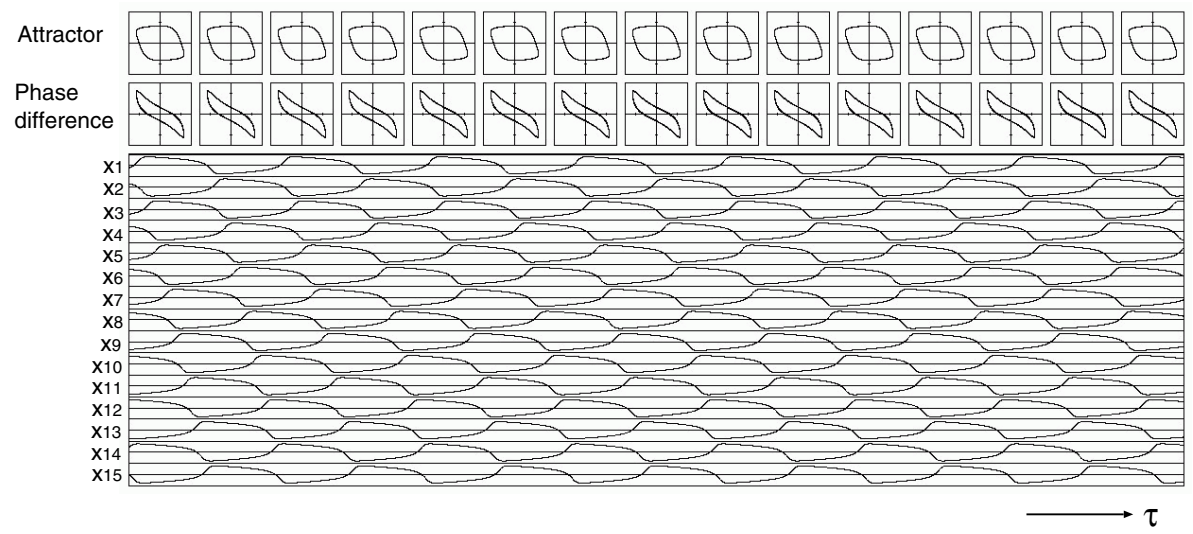

(b) 15-phase synchronization.

Fig. 10. Computer simulated result for $N=15 . \varepsilon=2.6, \omega=1.37, \gamma=(0.4$ or -0.12$), \eta=0.01$. Upper figures: $x_{k}$ vs $y_{a k}+y_{b k}$. Middle figures: $x_{k}$ vs $x_{(k+1)}$. Lower figures: $\tau$ vs $x_{k} . k=1,2,3, \ldots, 15$.

[3] Ch. von der Malsburg and W. Schneider, "A neural cooktail-party processor," Biol. Cybern., vol.54, pp.29-40, 1986.

[4] Ch. von der Malsbuurg and J. Buhmann, "Sensory segmentation with coupled neural oscillators," Biol. Cybern., vol.67, pp.233-242, 1992.

[5] L.L. Bonilla, C.J. Perez Vicente and R. Spigler, "Time-periodic phases in populations of nonlinearly coupled oscillators with bimodal frequency distributions," Physica D: Nonlinear Phenomena, vol.113, no.1, pp.79-97, Feb. 1998.

[6] J.A. Sherratt, "Invading wave fronts and their oscillatory wakes are linked by a modulated traveling phase resetting wave," Physica D: Nonlinear Phenomena, vol.117, no.1-4, pp.145-166, June 1998.

[7] G. Abramson, V.M. Kenkre and A.R. Bishop, "Analytic solutions for nonlinear waves in coupled reacting systems," Physica A: Statistical Mechanics and its Applications, vol.305, no.3-4, pp.427-436, Mar. 2002.

[8] I. Belykh, M. Hasler, M. Lauret and H. Nijmeijer, "Synchronization and graph topology," Int. J. Bifurcation and Chaos, vol.15, no.11, pp.34233433, Nov. 2005.

[9] C.M. Gray, "Synchronous oscillations in neural systems: mechanisms and functions," J. Computational Neuroscience, vol.1, pp.11-38, 1994.

[10] J. Cosp, J. Madrenas, E. Alarcon, E. Vidal and G. Villar, "Synchronization of nonlinear electronic oscillators for neural computation," IEEE Trans. Neural Networks, vol.15, no.5, pp.1315-1327, Sep. 2004.

[11] T. Suezaki and S. Mori, "Mutual synchronization of two oscillators," Trans. IECE, vol.48, no.9, pp.1551-1557, Sep. 1965.

[12] H. Kimura and K. Mano, "Some properties of mutually synchronized oscillators coupled by resistance," Trans. IECE, vol.48, no.10, pp.16471656, Oct. 1965.

[13] T. Endo and S. Mori, "Mode analysis of a multimode ladder oscillator," IEEE Trans. Circuits Syst., vol.23, no.2, pp.100-113, Feb. 1976.
[14] T. Endo and S. Mori, "Mode analysis of a two-dimensional low-pass multimode oscillator," IEEE Trans. Circuits Syst., vol.23, no.9, pp.517530, Sep. 1976.

[15] T. Endo and S. Mori, "Mode analysis of a ring of a large number of mutually coupled van der Pol oscillators," IEEE Trans. Circuits Syst., vol.25, no.1, pp.7-18, Jan. 1978.

[16] S.P. Datardina and D.A. Linkens, "Multimode oscillations in mutually coupled van der Pol type oscillators with fifth-power nonlinear characteristics," IEEE Trans. Circuits Syst., vol.25, no.5, pp.308-315, May 1978.

[17] Y. Nishio and S. Mori, "Mutually coupled oscillators with an extremely large number of steady states," Proc. of ISCAS'92, vol.2, pp.819-822, May 1992.

[18] M. Yamauchi, M. Wada, Y. Nishio and A. Ushida, "Wave propagation phenomena of phase states in oscillators coupled by inductors as a ladder," IEICE Trans. Fundamentals, vol.E82-A, no.11, pp.2592-2598, Nov. 1999.

[19] M. Yamauchi, Y. Nishio and A. Ushida, "Phase-waves in a ladder of oscillators" IEICE Trans. Fundamentals, vol.E86-A, no.4, pp.891-899, Apr. 2003.

[20] B. Liu, X. Liu, G. Chen and H. Wang, "Robust impulsive synchronization of uncertain dynamical networks," IEEE Trans. Circuits Syst. $I$, vol.52, no.7, pp.1431-1441, July 2005.

[21] Y. Nishio and S. Mori, "Chaotic phenomena in nonlinear circuits with time-varying resistors," IEICE Trans. Fundamentals, vol.E76-A, no.3, pp.467-475, Mar. 1993. 


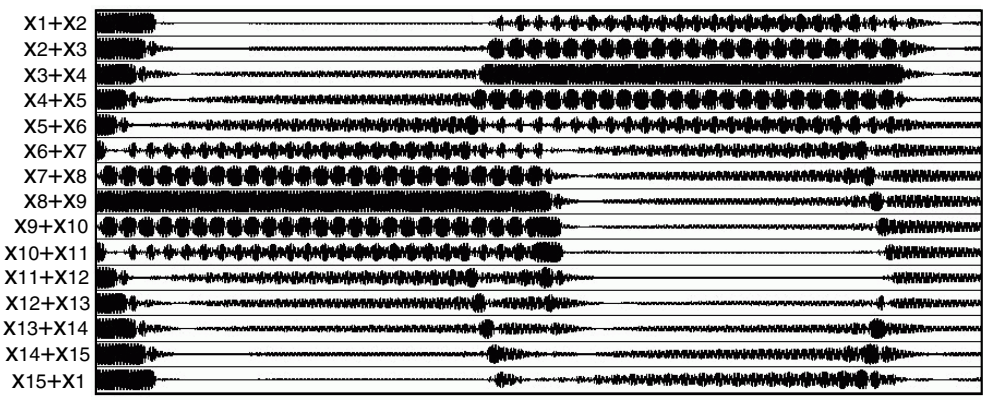

Fig. 11. Wave propagation phenomena. $\varepsilon=2.6, \omega=1.37, \gamma=(0.4$ or -0.12$), \eta=0.01$

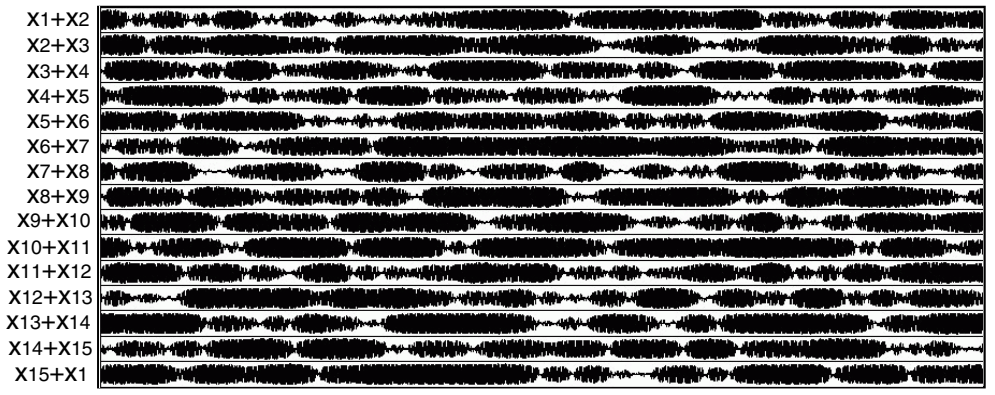

(a) $\varepsilon=2.6, \omega=0.87, \gamma=(0.2$ or -0.2$), \eta=0.01$.

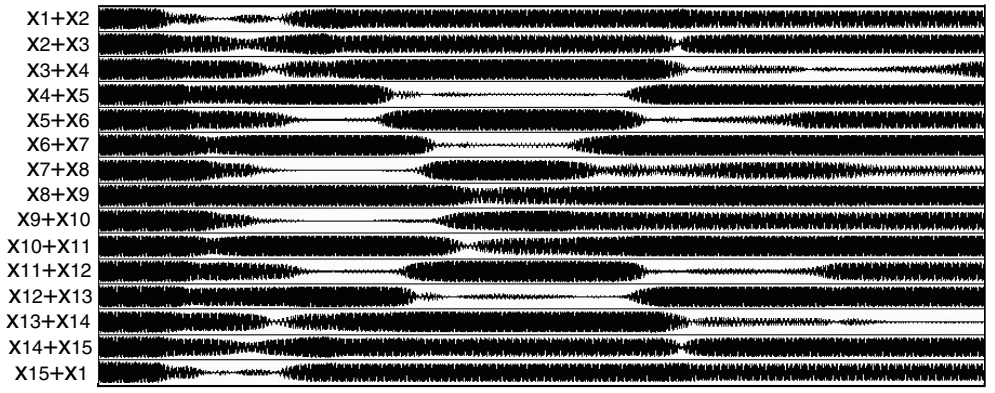

(b) $\varepsilon=2.6, \omega=1.00, \gamma=(0.2$ or -0.2$), \eta=0.01$.

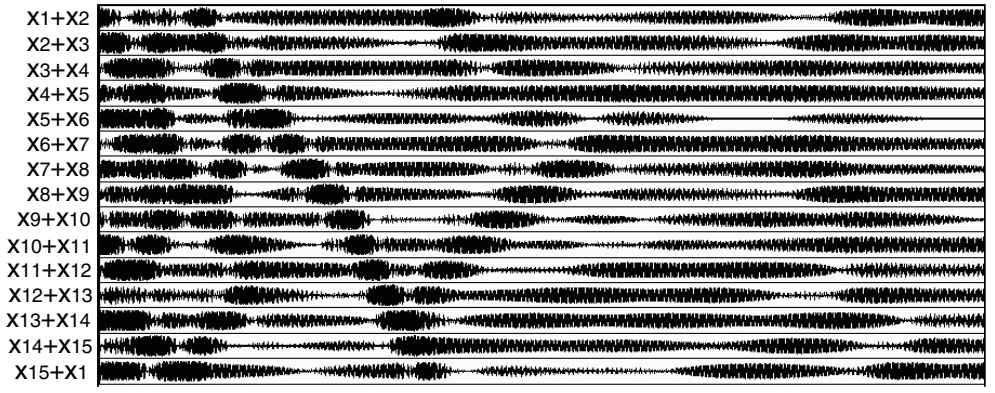

$\mathrm{X} 15+\mathrm{X} 1 \mathrm{~T}$

(c) $\varepsilon=2.6, \omega=2.52, \gamma=(0.2$ or -0.2$), \eta=0.01$.

Fig. 12. Complex phenomena 